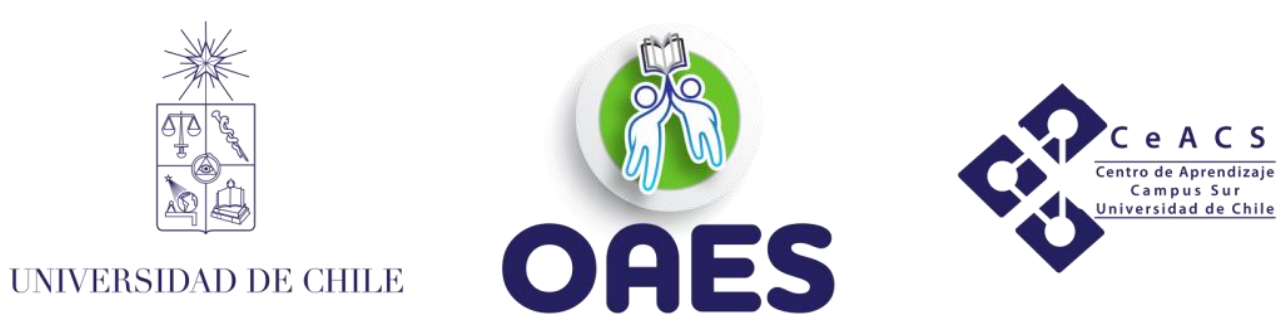

\title{
Balance de Carga Metodológico. Análisis en el primer nivel de Técnico Universitario en Administración, Universidad Autónoma de Chile, sede Temuco.
}

Mg. Luis Edmundo Donaire Herrera / luis.donaire@uautonoma.cl Mg. Alfredo Oyarzún Gutiérrez / alfredo.oyarzun@uautonoma.cl Mg. Jorge Osvaldo Rojos Torres / jorge.rojos@uautonoma.cl Mg. Cristian Barrientos González / cristian.barrientos@uautonoma.cl Dr. José Luis Saavedra Lucero / jose.saavedra@uautonoma.cl

Línea temática: Innovaciones curriculares para el aprendizaje

\section{Resumen:}

La gestión docente en las comunidades académicas permite diseñar estrategias pedagógicas colaborativas entre docentes. En este contexto, el balancear la carga académica de los estudiantes es una gestión tendiente al logro de resultados de aprendizaje.

Ante bajos rendimientos académicos de los estudiantes, el determinar el impacto del balance de carga metodológico en los resultados de aprendizaje es un objetivo a lograr, lo que justifica esta estrategia caracterizada por la diversidad de metodologías de aprendizaje aplicadas por los docentes del mismo nivel.

A partir de un diagnóstico de los estudiantes de la cohorte 2018, de la carrera de Técnico Universitario en Administración de la Universidad Autónoma de Chile, sede Temuco, se aplicó el balance de carga metodológico; el procedimiento evalúa las metodologías planificadas por los docentes en forma integrada, con el fin de reorientar la implementación de metodologías activo participativas, y finalmente, tender a lograr las competencias contempladas en el perfil de egreso de la carrera.

Se logra mejorar los resultados aprendizajes, promediando un aumento de un 36, $6 \%$ en los niveles de "destacado" en las evaluaciones finales de las asignaturas y bajando de un 20,7 a un 9,3\% en los niveles" insuficiente" en los promedios finales del nivel.

Palabras claves: balance de carga, innovación pedagógica, metodologías y aprendizaje.

\section{Introducción y antecedentes:}

El desarrollo de competencias en los estudiantes implica generar en éstos las capacidades de "movilizar un conjunto de recursos cognoscitivos, conocimiento, capacidades, información, etc., para enfrentar con pertinencia y eficacia a una familia de situaciones" (Perrenoud, 2000, p. 19). Si bien una capacidad se apoya en los conocimientos, pero no se reduce exclusivamente al uso racional de ellos o a la sola aplicación de procedimientos aprendidos, por lo que la formación universitaria debe alejarse del sólo traspaso de contenidos verbalizados informados en tediosas clases teóricas frontales y conductistas, para trasladarse al campo del aprendizaje contextualizado, dinámico y acompañado del hacer, única forma de desarrollar competencias en las dimensiones de conocimiento, habilidades y actitudes planteadas por Perrenoud. La generación de competencias debe estar acompañada, 


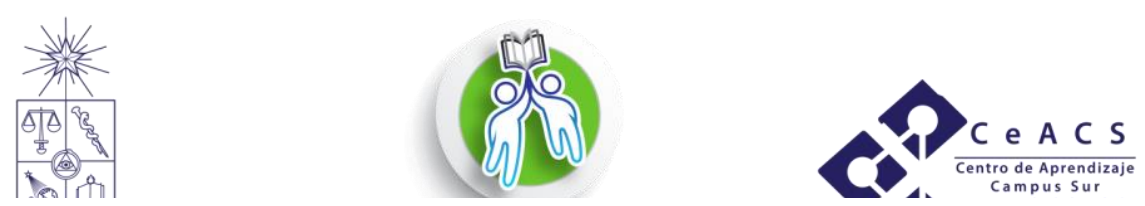

entonces, de estrategias metodológicas que promuevan el aprendizaje activo, donde los estudiantes auto gestionen sus aprendizajes, desarrollando su autonomía y el pensamiento crítico. Según Zabalza (2002), las demandas de la sociedad para los profesionales del futuro implica responder a las exigencias de la empleabilidad, caracterizado por la calidad, movilidad y capacidad de cambio, además de mejoras en la gestión de recursos e incorporación de nuevas tecnologías, por lo que la formación en la educación terciaria debe tener como objetivo el desarrollo de una enseñanza orientada a la generación de competencias socialmente demandadas, para responder a un escenario futuro dinámico y competitivo.

Este estudio se realiza sobre la base a los antecedentes de planificación docente sistematizados en una plataforma informática, analizando la información de las cinco semanas del mes de mayo 2018 (126 bloques u horas pedagógicas de clase) y junio del mismo semestre. En esta plataforma es posible almacenar información clase a clase a partir de las metodológicas de enseñanza y las estrategias evaluativas para cada asignatura del nivel.

\section{Desarrollo del trabajo y/o metodología:}

El público objetivo de estudio está conformado por 59 estudiantes de primer nivel de la cohorte 2018, de la carrera de Técnico Universitario en Administración de la Universidad Autónoma de Chile, sede Temuco. El estudio se realiza en el contexto de la comunidad académica de la carrera del primer nivel, integrada por sus estudiantes, equipo directivo y ocho docentes, cuatro de la planta académica y cuatro de jornada part time.

Las comunidades académicas comprenden un trabajo pedagógico colaborativo e integrado organizado con docentes, donde las estrategias pedagógicas globales e integradas mejoran los niveles de resultados de aprendizajes de los estudiantes. El objetivo general es favorecer a los estudiantes en la integración de competencias en desarrollo propias de su carrera. Se utiliza plataformas informáticas para el análisis de perfiles de ingreso y acompañamiento pedagógico, en donde el balance de carga de evaluaciones y metodologías es un producto de la comunidad educativa.

El Balance de carga es un procedimiento que permite evaluar las metodologías y estrategias evaluativas utilizadas por los docentes según la organización semanal, con el fin de orientar la utilización de metodologías activo participativas que permitan romper con la monotonía y predominancia de las actividades conductistas y clases frontales en el aula.

La primera evaluación regular del público objetivo generó en promedio un $22,3 \%$ de evaluaciones en el nivel de destacado, cuya calificación es mayor a 6,0 y un 20,7\% de estudiantes en nivel "insuficiente" en las asignaturas: Administración, Contabilidad, Introducción a las matemáticas, Habilitación profesional y Electivo de comunicación.

En este contexto, a partir del análisis de la información de la planificación docente extraídos de la plataforma en mayo 2018, la comunidad académica propone capacitar a sus docentes, reorganizando las actividades y estrategias metodológicas aplicadas en aula, con el fin de aliviar y facilitar el balance de carga metodológico en los estudiantes, utilizando otras herramientas como por ejemplo aprendizajes basado en proyectos, estudios de casos, etc. La plataforma institucional permite almacenar información de la planificación docente para balancear la carga académica. Se proyecta que una mejora significativa en la organización docente impactará en el logro de los aprendizajes de los estudiantes. 


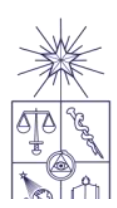

UNIVERSIDAD DE CHILE

\section{Resultados:}

La Tabla 1 muestra los resultados de la primera evaluación aplicada en abril de 2018, distribuidos en niveles de logro. Se aprecia un $22,3 \%$ en el nivel de destacado y un $20,7 \%$ en promedio en nivel insuficiente. Esta situación inicial justificó el análisis del balance de carga metodológico.

Tabla 1

Evaluaciones iniciales, abril 2018.

\begin{tabular}{lcccc}
\hline Asignatura & $\begin{array}{c}\text { Destacado (\%) } \\
(>\mathbf{6 , 0}-\mathbf{7 , 0})\end{array}$ & $\begin{array}{c}\text { Habilitado (\%) } \\
(>\mathbf{5 , 0}-\mathbf{6 , 0 )}\end{array}$ & $\begin{array}{c}\text { Regular (\%) } \\
\mathbf{( 4 , 0 - 5 , 0 )}\end{array}$ & $\begin{array}{c}\text { Insuficiente (\%) } \\
(<\mathbf{4 , 0 )}\end{array}$ \\
\hline Administración & 8,1 & 27,4 & 29,0 & 35,5 \\
Contabilidad & 32,3 & 35,5 & 16,1 & 16,1 \\
Matemática & 23,8 & 31,7 & 15,9 & 28,6 \\
Habilitación & 9,4 & 35,9 & 34,4 & 20,3 \\
Comunicación & 38,2 & 55,9 & 2,9 & 2,9 \\
\hline Promedio & $\mathbf{2 2 , 3}$ & $\mathbf{3 7 , 3}$ & $\mathbf{1 9 , 7}$ & $\mathbf{2 0 , 7}$ \\
\hline
\end{tabular}

La Tabla 2 muestra el balance de carga metodológico del mes de mayo de 2018, el cual se caracterizó por una mayor frecuencia de estrategias metodológicas conductistas "clase teórica", ponderando un $68 \%$ del total de las horas del nivel, y en donde sólo un 32\% del total participaban estrategias activo participativas, no existiendo equilibrio y evidenciándose falta de balance de carga metodológico.

Tabla 2

Balance de carga planificación inicial, periodo mayo 2018.

\begin{tabular}{lccc}
\hline $\begin{array}{l}\text { Metodología de enseñanza } \\
\text { aprendizaje }\end{array}$ & $\begin{array}{c}\text { Bloque (hora } \\
\text { pedagógica) }\end{array}$ & $\begin{array}{l}\text { Ponderación } \\
(\boldsymbol{\%})\end{array}$ & $\begin{array}{l}\text { Resumen } \\
(\boldsymbol{\%})\end{array}$ \\
\hline Clase teórica con ejercicios & 51 & 40 & 68 \\
Clase teórica expositiva & 35 & 28 & 32 \\
\hline Taller & 32 & 25 & $\mathbf{1 0 0}$ \\
Laboratorio & 8 & 6 & $\mathbf{1 0 0}$ \\
\hline Totales & $\mathbf{1 2 6}$ & & \\
\hline
\end{tabular}

Luego del trabajo de análisis del balance de carga se logra intencionar una mayor diversificación y equilibrio de las actividades de enseñanza y aprendizaje, en donde las clases teóricas bajan a un 46 $\%$, y los talleres y laboratorios suben a un $53 \%$ del total de horas (Tabla 3). Se logra evidenciar un equilibrio respondiendo a una mayor diversidad de ritmos de aprendizaje de los estudiantes. 
Tabla 3

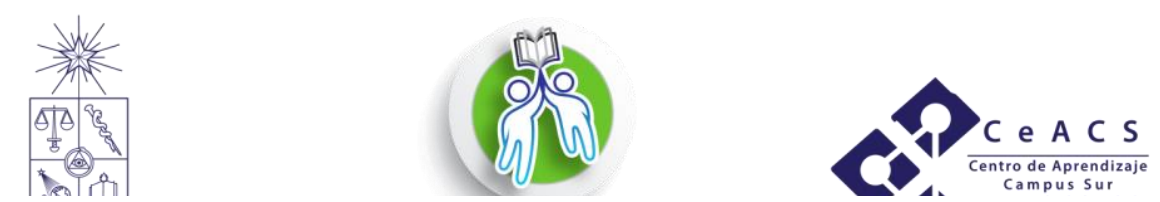

Balance de carga planificación inicial, periodo junio 2018.

\begin{tabular}{lccc}
\hline $\begin{array}{l}\text { Metodología de enseñanza } \\
\text { aprendizaje }\end{array}$ & $\begin{array}{c}\text { Bloque (hora } \\
\text { pedagógica) }\end{array}$ & $\begin{array}{l}\text { Ponderación } \\
(\boldsymbol{\%})\end{array}$ & $\begin{array}{l}\text { Resumen } \\
(\boldsymbol{\%})\end{array}$ \\
\hline Clase teórica con ejercicios & 46 & 29 & 46 \\
Clase teórica expositiva & 28 & 18 & 53 \\
\hline Taller & 65 & 41 & 1 \\
Laboratorio & 19 & 12 & $\mathbf{1 0 0}$ \\
\hline Trabajo aula virtual & 2 & 1 & $\mathbf{1 0 0}$ \\
\hline Totales & $\mathbf{1 6 0}$ & &
\end{tabular}

La Tabla 4 muestra los rendimientos académicos a final de semestre, llegando a un 58,9\% las evaluaciones promedio en la categoría de "destacado" y bajando a un 9,3\% el nivel de insuficiente.

Tabla 4

Evaluaciones finales, julio 2018.

\begin{tabular}{lcccc}
\hline Asignatura & $\begin{array}{c}\text { Destacado (\%) } \\
(>\mathbf{6 , 0} \mathbf{- 7 , 0 )}\end{array}$ & $\begin{array}{c}\text { Habilitado (\%) } \\
(>\mathbf{5 , 0}-\mathbf{6 , 0 )}\end{array}$ & $\begin{array}{c}\text { Regular (\%) } \\
(\mathbf{4 , 0}-\mathbf{5 , 0 )}\end{array}$ & $\begin{array}{c}\text { Insuficiente (\%) } \\
(<\mathbf{4 , 0})\end{array}$ \\
\hline Administración & 67,7 & 27,4 & 3,2 & 1,7 \\
Contabilidad & 56,5 & 24,2 & 9,7 & 9,7 \\
Matemática & 52,4 & 30,2 & 7,9 & 9,5 \\
Habilitación & 56,3 & 25,0 & 7,8 & 10.9 \\
Comunicación & 61,8 & 14,7 & 8,8 & 14,7 \\
\hline Promedio & $\mathbf{5 8 , 9}$ & $\mathbf{2 4 , 3}$ & $\mathbf{7 , 5}$ & $\mathbf{9 , 3}$ \\
\hline
\end{tabular}

Finalmente, existe una alta satisfacción en los docentes; todos señalaron que su participación fue activa en la comunidad académica, en las decisiones de gestión pedagógica y en el balance de carga metodológico. Indicaron sentirse motivados al intercambiar buenas prácticas con sus pares, concluyendo que las diversas estrategias pedagógicas impactaron positivamente en los resultados de aprendizaje de los estudiantes.

El $86 \%$ de los estudiantes señalaron alta satisfacción al haber logrado sus aprendizajes, sintiéndose motivados en las actividades y reconociendo colaboración entre sus docentes.

\section{Discusión y conclusiones:}

Es posible evidenciar en el estudio realizado durante los meses de mayo y junio del 2018, una significativa mejora en la gestión pedagógica global de la comunidad académica del primer nivel de la carrera de Técnico Universitario en Administración, adecuando el balance de carga metodológico para los estudiantes del nivel. Esta mejora ha facilitado el logro de los aprendizajes en cada una de las asignaturas, aumentando progresivamente en un 36,6 \% los niveles de "destacado" en las evaluaciones finales del semestre respecto a las evaluaciones iniciales, y bajando los niveles de insuficiente de un $20,7 \%$ a un $9,3 \%$ como promedios finales. 
A través de la utilización de una herramienta informática para la gestión del aula, se hizo partícipes a los docentes de las asignaturas trabajando en forma colaborativa e integrada. Se logró la implementación de las distintas estrategias metodológicas activas participativas centradas en el estudiante propuestas por Zabalza. Todos los docentes tienen un nivel de percepción alta en términos de haber alcanzado un trabajo colaborativo e integrado el cual impactó en el logro de mejoras en los rendimientos académicos de los estudiantes. A su vez, los estudiantes manifestaron altos niveles de satisfacción reconociendo a las estrategias pedagógicas globales como causales de sus mejoras en los aprendizajes de las distintas asignaturas del nivel.

El resultado del balance carga metodológico fue diverso y equilibrado, el cual reorientó la forma de organización docente, para romper con la monotonía de las actividades en el aula, con el fin último de aliviar y facilitar la carga de trabajo a los estudiantes en el tránsito del aprendizaje y permitir el logro de las competencias con las dimensiones propuestas por Perrenoud.

\section{Bibliografía:}

Perrenoud, P. (2000). El arte de construir competencias. Barcelona, España: Editorial Graó.

Zabalza, M. (2002). La Enseñanza universitaria. El escenario y sus protagonistas. Madrid, España: Ed. Narcea S.A De Ediciones. 